\title{
Multiple sclerosis in Algeria, a multidisciplinary management at Bejaia University Hospital Center.
}

\author{
F. Bouali ${ }^{\text {a }}$ S. Khellaf ${ }^{\mathrm{a}}$, N. Saaoui ${ }^{\mathrm{a}}$, H. Meziani ${ }^{\mathrm{a}}$, D. Si Ahmed ${ }^{\mathrm{b}}$ \\ a boualifaycal@hotmail.fr and saddek.khellaf@gmail.com \\ ${ }^{a}$ Internal Medicine Department, Bejaia University Hospital Center, Bejaia 06000, Algeria \\ ${ }^{b}$ Internal medicine Department, Mustapha Pacha University Hospital Center, Algiers 16000, Algeria
}

\begin{abstract}
The aim of our study is to establish the clinical, para-clinical, therapeutic and progressive profile of Multiple sclerosis (MS) in the Algerian region of Bejaia. We performed a retrospective descriptive study for a period of 4 years, going from January 2015 to December 2018. Diagnosis of MS was retained according to the 2010 McDonald criteria. Epidemiological characteristics were studied, such as first clinical presentation, clinical form, biological and neuroradiological data, current therapeutic management as well as evolutionary profile by evaluation of the Expanded Disability Status Scale (EDSS). We collected 109 Patient with MS, including $71(65 \%)$ women and $38(35 \%)$ men with a Sex Ratio F / M of 1.86. Average age of patients was 38.7 years with extremes between 17 and 65 years. Average age of onset was 31 years (ranging from 11 to 53 years). MS diagnostic delay was 2.9 years. The most frequent MS form was the relapsing remitting $(80 \%)$, then the secondary progressive $(12 \%)$ and finally the primary progressive form in $8 \%$ of cases. Patients received relapses corticosteroid treatment in $73 \%$ of cases. Disease modifying therapies (DMT) was prescribed based on interferon beta in 83 patients, Natalizumab in 2 patients and cyclophosphamide in 22 patients. We observed that EDSS increased from less than 5 in all patients at onset to more than 5 in $11 \%$ of patients after an average period of 6.7 years. Despite the small sample of our population, the severe aspect of North African MS could be clearly seen in our study.
\end{abstract}

Published by IJRP.ORG. Selection and/or peer-review under responsibility of International Journal of Research Publications (IJRP.ORG)

\section{Introduction}

Multiple sclerosis (MS) is a chronic inflammatory demyelinating and degenerative disease of central nervous system (CNS), resulting sometimes by severe handicap in young people [1]. Signs and disease symptoms can occur individually or in combination, thus in an acute or progressive mode. The two useful elements in practice are the onset mode (by relapses or progressing) and the present disability status. This will distinguish 3 different forms, the relapsing remitting (RR) form and the progressive forms. These progressive forms occur either immediately at the beginning of the disease, then called primary progressive MS (PP), or following the development of a RR form, these are secondary progressive MS (SP) [2-4]. 
Diagnosis of MS, defined by Poser [5] then McDonald [6], is based on some criteria of spatial and temporal dissemination of the lesions. These criteria have been revised several times, the last one in 2017 [7]. Predilection exam remains MRI [8-10], whether or not supplemented by a lab prove of intrathecal secretion of immunoglobulins [11]. Pathophysiologic mechanisms of MS remain poorly understood, but it is generally accepted that it develops in subjects with individual genetic susceptibility who are subsequently confronted with various environmental factors [12-14]. This initiates an immune reaction $[1,15]$ directed against the CNS at the origin of MS various symptoms [16].

Prevalence of multiple sclerosis in the world is variable and corresponds to a north-south gradient in each hemisphere [17, 18], epidemiological data find prevalence around 20/100 000 in Central and Latin America, 60 in Australia, 150 and 205 cases in northern European countries, or even up to 357 cases in Canada. The prevalence seems to be very low in Japan (13/100 000) and in China [19-21]. Worldwide incidence of the disease has increased over the 50 past years [22], this increase seems to be faster in women than in men [2325]. Prevalence and incidence of MS are not well documented in many North African and Middle Eastern (MENA) countries including Algeria, and most prevalence estimates come from isolated hospital studies [26].

In Algeria, the reported prevalence, from studies carried out in Tlemcen [27] then in Blida [28], is estimated respectively at 23.7 and 39.7 cases per 100,000 inhabitants. As for Bejaia, no study has been carried out on MS, so we deemed it appropriate to study the clinical, para-clinical, therapeutic and progressive profile of this disease in the region.

\section{Research elaborations}

This is a descriptive retrospective study, which took place in the department of internal medicine at the Khelil Amrane university hospital center in Bejaia, in eastern Algeria. The study was carried out over a period of 4 years, from January 2015 to December 2018. Diagnosis of MS was retained according to the 2010 McDonald criteria. Epidemiological characteristics were studied, such as first clinical presentation, clinical form, biological and neuroradiological data, current therapeutic management as well as follow-up profile by assessing the Expanded Disability Status Scale (EDSS). Statistical analysis was performed using IBM Statistical Package for Social Sciences (SPSS) version 20.0 (Chicago, IL, USA).

\section{Results}

As shown in Table 1, we collected 109 PwMS, including $71(65 \%)$ women and $38(35 \%)$ men with a F/M Sex Ratio of 1.86. Average age of patients was 38.7 years with extremes between 17 and 65 years. Average age of onset ranging from 11 to 53 years was 31 years. Most frequent first symptoms were motor deficit (in 45 cases) and reduced visual acuity (in 22 cases). Brain magnetic resonance imaging (MRI) scan was performed in all patients and found demyelinating lesions in characteristic MS areas as the following proportions; periventricular region in $74(67.9 \%)$, juxtacortical region in $90(82.6 \%)$, posterior fossa in $54(49.4 \%)$ and spinal cord in $40(36.7 \%)$ patients. Lumbar puncture (PL) was performed in $79 \%$ of patients and immunological study of the cerebrospinal fluid (CSF) showed intra thecal immunoglobulin synthesis in 66 (76\%) cases. Average diagnostic delay was 2.9 years. The most frequent MS form was the relapsing remitting $(80 \%)$, then the secondary progressive $(12 \%)$ and finally the primary progressive form in $8 \%$ of cases. We observed that initial EDSS results were already higher than 3.5 in $17 \%$ of our patients. After an average evolution period of 6.7 years, this score increased from less than 5 in all patients to more than 5 in $11 \%$ of patients. Patients received relapses treatment with corticosteroids in $73 \%$ of cases. Disease modifying 
therapies (DMT) was prescribed based on beta interferon in $83(76.1 \%)$ patients, Natalizumab in 2 patients and cyclophosphamide in $22(20.1 \%)$ patients.

Table 1. Characteristics of the study population.

\begin{tabular}{ll}
\hline & $\mathrm{N}(\%)$ \\
\hline Average age (years) ${ }^{*}$ & $38.7[17-65]$ \\
Women & $71(65 \%)$ \\
Men & $38(35 \%)$ \\
Sex-Ratio F / M & 1.86 \\
Average age of onset & $31[11-53]$ \\
Initial clinical presentation & \\
$\quad$ Motor deficit & $45(49 \%)$ \\
$\quad$ Decreased visual acuity & $22(24 \%)$ \\
$\quad$ Sensitive disorders & $19(21 \%)$ \\
$\quad$ Gait disorders & $9(10 \%)$ \\
Average diagnostic times (year) & 2.9 \\
Clinical form: & \\
$\quad$ Relapsing Remitting & $88(80 \%)$ \\
$\quad$ Secondary Progressive & $13(12 \%)$ \\
$\quad$ Primary progressive & $8(8 \%)$ \\
Brain MRI scan & $109(100 \%)$ \\
Lumbar puncture & $86(79 \%)$ \\
Intrathecal IgG synthesis & $66(71 \%)$ \\
Initial EDSS: & \\
0 to 1.5 &
\end{tabular}




\section{Conclusion}

In most of autoimmune diseases, women are affected more frequently than men [29]. Similar to the results of our study, several demographic studies have shown that the female prevalence in multiple sclerosis (MS) is almost constant [30]. This preponderance is further increased in early and late cases, in family cases as well as in twin pairs of MS [31]. This predominance could be attributed to environmental (vitamin D), hormonal, sexual or even genetic factors [30,32].

The age of onset of MS can vary from childhood to adulthood. In our study, it was 31 years old. Several studies [33-36] concerning the role of this age of onset in the later course of the disease, have reported that a higher age at onset is associated with a less favorable prognosis. However, other studies have not confirmed this result [37, 38]. Our PwMS present a wide variety of neurological symptoms, being linked to the fact that inflammatory manifestations can involve different structures of the CNS [35, 39].

Despite the small sample of our study, the severe aspect of North African MS could be seen. We found out that $11 \%$ of our patients lost their walking autonomy after an average evolution period of 6.7 years. this observation is added to many other studies results, like the multicenter study performed by Hechem et al., and report that MS in Algeria would have a severe course with a rapid disability onset [40]. Similar results have been observed in Morocco [41] as well as in studies carried out in Europe also on North African migrants [42].

MS in North Africa remains a particular severe form requiring broader genetic and environmental studies and well-coordinated multidisciplinary management to allow not only an accurate and early diagnosis but above all a less disabling course under a well targeted treatment.

\section{Conflicts of interest}

Authors of this manuscript do not declare any conflict of interest.

\section{References}

1. Compston, A. and A. Coles, Multiple sclerosis. Lancet, 2008. 372(9648): p. 1502-17.

2. Brochet, B., et al., Chapitre 1 - Signes et symptômes de la sclérose en plaques, in La Sclérose en Plaques - Clinique et Thérapeutique, B. Brochet, et al., Editors. 2017, Elsevier Masson: Paris. p. 3-78.

3. Feinstein, A., et al., Clinically isolated lesions of the type seen in multiple sclerosis: a cognitive, psychiatric, and MRI follow up study. J Neurol Neurosurg Psychiatry, 1992. 55(10): p. 869-76.

4. Filippi, M., et al., Changes in the normal appearing brain tissue and cognitive impairment in multiple sclerosis. J Neurol Neurosurg Psychiatry, 2000. 68(2): p. 157-61.

5. Poser, C.M., et al., New diagnostic criteria for multiple sclerosis: guidelines for research protocols. Annals of Neurology: Official Journal of the American Neurological Association and the Child Neurology Society, 1983. 13(3): p. 227-231.

6. McDonald, W.I., et al., Recommended diagnostic criteria for multiple sclerosis: guidelines from the International Panel on the diagnosis of multiple sclerosis. Annals of Neurology: Official Journal of the American Neurological Association and the Child Neurology Society, 2001. 50(1): p. 121-127.

7. Thompson, A.J., et al., Diagnosis of multiple sclerosis: 2017 revisions of the McDonald criteria. The Lancet Neurology, 2018. 17(2): p. 162-173. 
8. Fazekas, F., et al., The contribution of magnetic resonance imaging to the diagnosis of multiple sclerosis. Neurology, 1999. 53(3): p. 448-448.

9. Filippi, M., et al., MRI criteria for the diagnosis of multiple sclerosis: MAGNIMS consensus guidelines. The Lancet Neurology, 2016. 15(3): p. 292-303.

10. Cortese, R., et al., Advances in brain imaging in multiple sclerosis. Ther Adv Neurol Disord, 2019. 12: p. 1756286419859722.

11. Correale, J. and M.d.1.M.B. Molinas, Oligoclonal bands and antibody responses in multiple sclerosis. Journal of neurology, 2002. 249(4): p. 375-389.

12. Pantazou, V., M. Schluep, and R. Du Pasquier, Environmental factors in multiple sclerosis. Presse Med, 2015. 44(4 Pt 2): p. e113-20.

13. Pugliatti, M., et al., Environmental risk factors in multiple sclerosis. Acta Neurol Scand Suppl, 2008. 188: p. 34-40.

14. Ebers, G.C., Environmental factors and multiple sclerosis. Lancet Neurol, 2008. 7(3): p. 268-77.

15. Vermersch, P., Advances in the management of MS symptoms: recently proposed clinical management algorithms. Neurodegener Dis Manag, 2015. 5(6 Suppl): p. 23-6.

16. Brassat, D., [Pathophysiology of multiple sclerosis]. Presse Med, 2010. 39(3): p. 341-8.

17. Koch-Henriksen, N. and P.S. Sorensen, Why does the north-south gradient of incidence of multiple sclerosis seem to have disappeared on the Northern hemisphere? Journal of the neurological sciences, 2011. 311(1-2): p. 58-63.

18. Simpson, S., et al., Latitude is significantly associated with the prevalence of multiple sclerosis: a meta-analysis. Journal of Neurology, Neurosurgery \& Psychiatry, 2011. 82(10): p. 1132-1141.

19. Thibault Moreau, R.D.P., Sclérose en plaques. Editions John Libbey Eurotext ed, ed. Doin. 2017: John Libbey Eurotext.

20. Warren, S.A., L.W. Svenson, and K.G. Warren, Contribution of incidence to increasing prevalence of multiple sclerosis in Alberta, Canada. Mult Scler, 2008. 14(7): p. 872-9.

21. Hader, W.J. and I.M. Yee, Incidence and prevalence of multiple sclerosis in Saskatoon, Saskatchewan. Neurology, 2007. 69(12): p. 1224-9.

22. Barnett, M.H., et al., Progressive increase in incidence and prevalence of multiple sclerosis in Newcastle, Australia: a 35-year study. J Neurol Sci, 2003. 213(1-2): p. 1-6.

23. Orton, S.M., et al., Sex ratio of multiple sclerosis in Canada: a longitudinal study. Lancet Neurol, 2006. 5(11): p. 932-6.

24. Trojano, M., et al., Geographical variations in sex ratio trends over time in multiple sclerosis. PloS one, 2012. 7(10): p. e48078e48078.

25. Alonso, A. and M.A. Hernán, Temporal trends in the incidence of multiple sclerosis. A systematic review, 2008. 71(2): p. $129-135$.

26. Heydarpour, P., et al., Multiple Sclerosis Epidemiology in Middle East and North Africa: A Systematic Review and Meta-Analysis. Neuroepidemiology, 2015. 44(4): p. 232-44.

27. Z. Bedrane-Barka, prevalence, formes cliniques, evolution, et traitement de la sclerose en plaques dans la region de Tlemcen, in Thèse de DSM. 2013.

28. Drai, R., Etude clinique et Profil évolutif des patients suivis pour une Sclérose en plaques au niveau du CHU de Blida, avec une estimation de la prévalence dans la commune de Blida. , in Thèse de DSM,. 2018.

29. Beeson, P.B., Age and sex associations of 40 autoimmune diseases. Am J Med, 1994. 96(5): p. 457-62.

30. Milo, R. and E. Kahana, Multiple sclerosis: geoepidemiology, genetics and the environment. Autoimmun Rev, 2010. 9(5): p. A38794.

31. Duquette, P., et al., The increased susceptibility of women to multiple sclerosis. Can J Neurol Sci, 1992. 19(4): p. 466-71.

32. Sellner, J., et al., The increasing incidence and prevalence of female multiple sclerosis--a critical analysis of potential environmental factors. Autoimmun Rev, 2011. 10(8): p. 495-502.

33. Visscher, B.R., et al., Onset symptoms as predictors of mortality and disability in multiple sclerosis. Acta neurologica scandinavica, 1984. 70(5): p. 321-328.

34. Runmarker, B., et al., Prediction of outcome in multiple sclerosis based on multivariate models. Journal of neurology, 1994. 241(10): p. 597-604.

35. Weinshenker, B.G., Natural history of multiple sclerosis. Annals of Neurology: Official Journal of the American Neurological Association and the Child Neurology Society, 1994. 36(S1): p. S6-S11. 
36. Trojano, M., et al., Multivariate analysis of predictive factors of multiple sclerosis course with a validated method to assess clinical events. Journal of Neurology, Neurosurgery \& Psychiatry, 1995. 58(3): p. 300-306.

37. Moreau, T. and C. Confavreux, Can the prognosis of multiple sclerosis be predicted? Pathologie-biologie, 2000. $48(2)$ : p. $132-138$.

38. Ligouri, M., et al., Age at onset in multiple sclerosis. Neurological Sciences, 2000. 21(2): p. S825-S829.

39. Kister, I., et al., Natural history of multiple sclerosis symptoms. International journal of MS care, 2013. 15(3): p. 146-156.

40. Hecham, N., et al., Multiple sclerosis: progression rate and severity in a multicenter cohort from Algeria. Mult Scler, 2014. 20(14): p. 1923-4.

41. Araqi-Houssaini, A., et al., Multiple sclerosis severity score in a cohort of Moroccan patients. Mult Scler, 2014. 20(6): p. $764-5$.

42. Debouverie, M., et al., More severe disability of North Africans vs Europeans with multiple sclerosis in France. Neurology, 2007. 68(1): p. 29-32. 